\title{
Secure Logistic Regression Based on Homomorphic Encryption: Design and Evaluation
}

Miran Kim ${ }^{1}$, MSc, PhD; Yongsoo Song ${ }^{2,3}, \mathrm{PhD}$; Shuang Wang ${ }^{1}$, MSc, PhD; Yuhou Xia ${ }^{4}, \mathrm{MSc}$; Xiaoqian Jiang ${ }^{1}$, MSc, $\mathrm{PhD}$

${ }^{1}$ Division of Biomedical Informatics, University of California, San Diego, San Diego, CA, United States

${ }^{2}$ Department of Mathematical Sciences, Seoul National University, Seoul, Republic Of Korea

${ }^{3}$ Department of Computer Science and Engineering, University of California, San Diego, San Diego, CA, United States

${ }^{4}$ Department of Mathematics, Princeton University, Princeton, NJ, United States

\section{Corresponding Author:}

Miran Kim, MSc, PhD

Division of Biomedical Informatics

University of California, San Diego

9500 Gilman Drive

San Diego, CA, 92093

United States

Phone: 18582499978

Email: $\underline{\text { mrkim@ucsd.edu }}$

\section{Abstract}

Background: Learning a model without accessing raw data has been an intriguing idea to security and machine learning researchers for years. In an ideal setting, we want to encrypt sensitive data to store them on a commercial cloud and run certain analyses without ever decrypting the data to preserve privacy. Homomorphic encryption technique is a promising candidate for secure data outsourcing, but it is a very challenging task to support real-world machine learning tasks. Existing frameworks can only handle simplified cases with low-degree polynomials such as linear means classifier and linear discriminative analysis.

Objective: The goal of this study is to provide a practical support to the mainstream learning models (eg, logistic regression).

Methods: We adapted a novel homomorphic encryption scheme optimized for real numbers computation. We devised (1) the least squares approximation of the logistic function for accuracy and efficiency (ie, reduce computation cost) and (2) new packing and parallelization techniques.

Results: Using real-world datasets, we evaluated the performance of our model and demonstrated its feasibility in speed and memory consumption. For example, it took approximately 116 minutes to obtain the training model from the homomorphically encrypted Edinburgh dataset. In addition, it gives fairly accurate predictions on the testing dataset.

Conclusions: We present the first homomorphically encrypted logistic regression outsourcing model based on the critical observation that the precision loss of classification models is sufficiently small so that the decision plan stays still.

(JMIR Med Inform 2018;6(2):e19) doi: 10.2196/medinform.8805

\section{KEYWORDS}

homomorphic encryption; machine learning; logistic regression; gradient descent

\section{Introduction}

Biomedical data are highly sensitive and often contain important personal information about individuals. In the United States, health care data sharing is protected by the Health Insurance Portability and Accountability Act [1], whereas biomedical research practitioners are covered under federal regulation governing the "Common Rule," a federal policy that protects people who volunteer for federally funded research studies [2].
These policies set high standards on the protection of biomedical data and violations will lead to financial penalties and lost reputation. On the other hand, cloud computing, which significantly simplifies information technology environments, is the trend for data management and analysis. According to a recent study by Microsoft, nearly a third of organizations work with four or more cloud vendors [3]. The privacy concern, therefore, becomes a major hurdle for medical institutions to outsource data and computation to the commercial cloud. It is 
imperative to develop advanced mechanisms to assure the confidentiality of data to support secure analysis in the cloud environment.

An intuitive solution is to train a model without accessing the data and only obtain the estimated model parameters in a global manner. Assuming summary statistics can be shared, this can be done in a joint manner and we have developed the "grid logistic regression" [4-6] to show the feasibility of estimating the global parameters from distributed sources (eg, by only exchanging gradients and Hessian matrices). However, there are still vulnerabilities in sharing even the summary statistics; for example, the difference in mean age between a cohort of $n$ patients and another cohort of $n-1$ overlapped patients can reveal the actual age of a single patient.

Many medical decision-making systems rely on the logistic regression model [7-9]. However, to use them appropriately, we need to provide a sufficient sample, which requires a sample size calculation. Peduzzi et al [10] suggested a simple guideline for a minimum number of cases to include in the study: let $p$ be the smallest of the proportions of negative or positive cases in the population and $k$ the number of covariates (the number of independent variables), then the minimum number of cases to include is $N=10 \cdot k / p$. For example, one has three covariates to be included in the model and the proportion of positive cases in the population is $0.2(20 \%)$. The minimum number of cases required is $10 \cdot 3 / 0.20=150$. For rare disease studies with many variables, it is even harder to collect enough samples from a single institution to meet this goal. We need to circumvent the privacy barriers to feed the model with more samples from different sources. As shown in Figure 1, homomorphic encryption techniques can support typical secure computations (eg, secure outsourcing and secure multiparty computation) and mitigate the privacy risks by allowing all computation to be done in the encrypted format.
Graepel et al [11] shed light on machine learning with homomorphically encrypted data. The article discussed scenarios that are appropriate and inappropriate to exercise machine learning with homomorphic encryption techniques. The authors provided two examples: linear means classifier and linear discriminative analysis, which can be achieved by using low-degree polynomials in homomorphic encryption. However, these simple parametric models do not handle complex datasets well and they do not represent the mainstream machine learning technologies used in biomedical research $[12,13]$. Additional work was carried out by Bos et al [14] to demonstrate the feasibility of making a prediction on encrypted medical data in Microsoft's Azure cloud. However, instead of learning from the data, this model only makes predictions using learned logistic regression models in a privacy-preserving manner. Similarly, a more recent work called CryptoNets applied trained neural networks to encrypted data only for the evaluation purpose [15]. Related works are summarized in Table 1.

In the current literature, most similar to our work are Aono et al [16] and Mohassel et al [17], but they are also very different from ours in assumptions and methods. Aono et al introduced an approximation to convert the likelihood function into a low-degree polynomial and used an additive homomorphic encryption to aggregate some intermediary statistics [16]. However, their scenario relies on the client to decrypt these intermediary statistics so that it can minimize the parameters locally. This is not a completely secure outsourcing scenario as ours, which works on encrypted data to obtain encrypted parameters without any client involvement. Mohassel et al developed secure two-party computation protocols to conduct the stochastic gradient descent for solving logistic regression and neural network problems [17].

Figure 1. Two secure models: (a) secure storage and computation outsourcing and (b) secure model outsourcing.
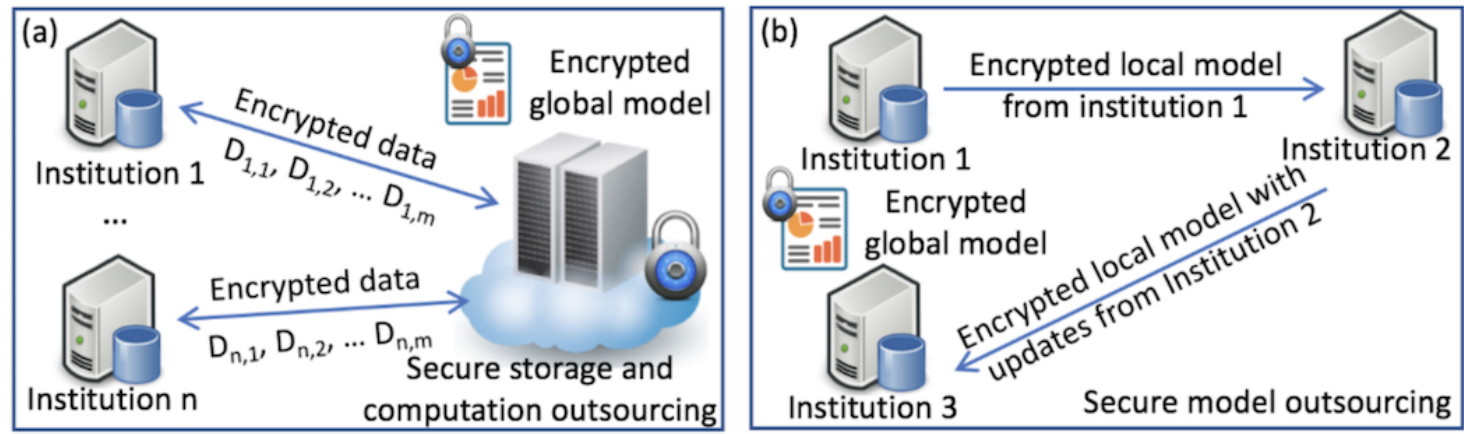
Table 1. Research works in secure analysis.

\begin{tabular}{lll}
\hline Reference & Problem & Techniques \\
\hline Graepel et al [11] & Linear means classifier/discriminative analysis & Homomorphic encryption \\
Bos et al [14] & Prediction using learned logistic regression model & Homomorphic encryption \\
Dowlin et al [15] & Prediction using learned neural networks & Homomorphic encryption \\
Aono et al [16] & Logistic regression & Multiparty computation \\
Mohassel et al [17] & Logistic regression & Homomomorphic encryption \\
This work & Logistic regression &
\end{tabular}

This method takes a completely different approach (garbled circuit and secret sharing vs homomorphic encryption) and the assumptions are widely different from ours (secure multiparty computation vs secure outsourcing). There are several prominent challenges related to scalability and efficiency. Traditional methods cannot handle many iterations of multiplications, which leads to a deep circuit and exponential growth in computational cost and storage size of the ciphertext. On the other hand, it is a nontrivial task to approximate certain critical functions in machine learning models using only low-degree polynomials. Naive approximation may lead to big errors and makes the solutions intractable. Our framework proposes novel methods to handle these challenges and makes it possible to learn a logistic regression model on encrypted data based completely on homomorphic encryption.

\section{Methods}

\section{Logistic Regression}

Logistic regression is a widely used learning model in biomedicine [13]. Data for supervised learning consist of pairs $\left(x_{i}, y_{i}\right)$ of a vector of covariates $x_{i}=\left(x_{i 1}, \ldots, x_{i \mathrm{~d}}\right)$ and a class label $y_{i}$ for $i=1, \ldots, n$. We assume that $y_{i}=1 /-1$ for binary classification. The model looks like:

$\operatorname{Pr}\left[y_{i} \mid x_{i}\right]=\sigma\left(y_{i} \cdot\left(1, x_{i}\right)^{\mathrm{T}} \beta\right)$

for the sigmoid function $\sigma(x)=1 /[1+\exp (-x)]$ where $\beta=\left(\beta_{0}\right.$, $\left.\beta_{1}, \ldots, \beta_{d}\right)$ are the model parameters to be estimated. Training methods of logistic regression aim to find the optimal parameters $\beta$, which minimizes the cost (negative log-likelihood)

$(1 / n) \Sigma_{1 \leq i \leq n} \log \left(1+\exp \left(-y_{i} \cdot\left(1, x_{i}\right)^{\mathrm{T}} \beta\right)\right)$

\section{Homomorphic Encryption for Approximate Arithmetic}

Homomorphic encryption is an encryption technique that allows computations on ciphertexts and generates encrypted results that match those of plaintext computation. We adopted a special cryptosystem developed by Cheon et al [18], which supports an approximate arithmetic of encrypted messages. Different from existing methods, this cryptosystem trades precision for efficiency so that the size of parameters does not grow too large (thus computationally feasible). Interested readers can refer to Multimedia Appendix 1 for more details. The cryptosystem supports key generation, encryption, decryption, addition, and multiplication operations. It also supports message packing and rotation, which are important to parallelize similar tasks.
A unique property of this cryptosystem is the following rescaling procedure, which plays an important role in controlling the magnitude of messages and, therefore, achieving the efficiency of approximate computation. The rescaling procedure coverts an encryption $c t$ of a message $m$ with a ciphertext modulus $q$ into an encryption $c t^{\prime}$ of $r^{-1} \cdot \mathrm{m}$ under the same secret key but a smaller modulus $q^{\prime}=r^{-1} \cdot q$, in which $r$ is a scaling factor. We denote the output ciphertext by $\mathrm{RS}(c t ; r)$. It enables us to round the message and reduce the size of significand by removing some inaccurate least significant bits as in the floating-point arithmetic. Informally, we will say that the input ciphertext modulus is reduced by $\log r$ bits after this procedure where the binary $\log$ arithm will be simply denoted by $\log (\cdot)$.

\section{Least Squares Approximation of the Sigmoid Function}

Unlike linear regression, logistic regression does not have a closed-form solution in most cases. As a result, we need to use nonlinear optimization methods to find the maximum likelihood estimators of the regression parameters. The Newton-Raphson [19] and the gradient descent [20] are the most commonly used methods for training. Because the Newton-Raphson method involves matrix inversion and most homomorphic encryption schemes do not naturally support division or matrix inversion, it is difficult to evaluate the method with homomorphic encryption schemes. On the other hand, gradient descent does not require the division operation and, therefore, it is a better candidate for homomorphically encrypted logistic regression. Thus, we choose the gradient descent algorithm as the training method for logistic regression.

Let $\left(x_{i}, y_{i}\right)$ be the supervised learning samples for $i=1, \ldots, n$. If we write $z_{i}=y_{\mathrm{i}} \cdot\left(1, x_{i}\right)$, the cost function for logistic regression is defined by:

$(1 / n) \Sigma_{1 \leq i \leq n} \log \left(1+\exp \left(-z_{i}^{\mathrm{T}} \beta\right)\right)$

Its gradient with respect to $\beta$ is computed by $-1 / n \Sigma_{1 \leq i \leq n} \sigma\left(-z_{i}^{\mathrm{T}} \beta\right) \cdot z_{i}$. To find a local minimum point, the gradient descent method updates the regression parameters using the following formula until $\beta$ converges:

$$
\beta \leftarrow \beta+(\alpha / n) \Sigma_{1 \leq i \leq n} \sigma\left(-z_{i}^{\mathrm{T}} \beta\right) \cdot z_{i},
$$

where $\alpha$ is the learning rate.

Although the gradient descent method seems better suited than other training methods for homomorphic evaluation, some technical problems remain for implementation. In the preceding update formula, the sigmoid function is the biggest obstacle for evaluation, since the existing homomorphic encryption schemes 
only allow evaluation of polynomial functions. Hence, the Taylor polynomials $T_{\mathrm{d}}(x)=\Sigma_{0 \leq k \leq d}\left(f^{(\mathrm{k})}(0) / k !\right) \cdot x^{k}$ have been commonly used for approximation of the sigmoid function [14,17]:

$$
\begin{aligned}
& \sigma(x)=1 / 2+(1 / 4) x-(1 / 48) x^{3}+(1 / 480) x^{5}-(17 / 80,640) \\
& x^{7}+(31 / 141,520) x^{9}+\mathrm{O}\left(x^{11}\right)
\end{aligned}
$$

However, we observed the input values $z_{i}^{\mathrm{T}} \beta$ of the sigmoid function during iterations on real-world datasets and concluded that the Taylor polynomial $T_{9}(x)$ of degree 9 is still not enough to obtain the desired accuracy (see Figure 2a). The size of error grows rapidly as $|x|$ increases. For instance, we have $T_{9}(4) \approx 4.44, T_{9}(6) \approx 31.23$, and $T_{9}(8) \approx 138.12$. In addition, we have to use a higher degree Taylor polynomial to guarantee the accuracy of regression, but it requires too many homomorphic multiplications to be practically implemented. In summary, the Taylor polynomial is not a good candidate for approximation because it is a local approximation near a certain point. Therefore, we adopted a global approximation method that minimizes the mean squared error (MSE). For an integrable function $f$, its mean square over an interval $I$ is defined by $(1 /|I|) \int_{I} f(x)^{2} \mathrm{~d} x$, where $|I|$ denotes the length of $I$. The least squares method aims to find a polynomial $g(x)$ of degree $d$ which minimizes the MSE $(1 /|I|) \int_{I}(f(x)-g(x))^{2} \mathrm{~d} x$. The least squares approximation has a closed formula that can be efficiently calculated using linear algebra.

In our implementation, we used the degree 3 and 7 least squares approximations of the sigmoid function over the interval $[-8,8]$, which contains all of the input values $\left(-z_{i}^{\mathrm{T}} \beta\right)$ during iterations. The least squares polynomials are computed as:

$$
\begin{aligned}
& g_{3}(x)=0.5+a_{1}(x / 8)+a_{3}(x / 8)^{3}, \\
& g_{7}(x)=0.5+b_{1}(x / 8)+b_{3}(x / 8)^{3}+b_{5}(x / 8)^{5}+b_{7}(x / 8)^{7},
\end{aligned}
$$

where the coefficients vectors are $\left(a_{1}, a_{3}\right) \approx(1.20096,-0.81562)$ and $\left(b_{1}, b_{3}, b_{5}, b_{7}\right) \approx(1.73496,-4.19407,5.43402,-2.50739)$. The degree 3 least squares approximation requires a smaller depth for evaluation, whereas the degree 7 polynomial has a better precision (see Figure 2b).

\section{Homomorphic Evaluation of Gradient Descent Algorithm}

We will describe how to encode data and explain how to analyze logistic regression on encrypted data. To speed up the computation, we will use the packing mechanism to batch $n$ slots and perform $n$ evaluations in parallel, where $n$ is the number of training data samples.

We start with a useful aggregation operation across plaintext slots from the literature [21-23]. Specifically, given a ciphertext representing a plaintext vector $\left(m_{1}, m_{2}, \ldots, m_{\mathrm{k}}\right)$, we introduce an algorithm (denoted by AllSum) that generates a ciphertext representing a value of $\Sigma_{1 \leq i \leq k} m_{\mathrm{i}}$ in each plaintext slot. Assume that $k$ is chosen as a power-of-two integer. The cyclic rotation by one unit produces a ciphertext encrypting the plaintext vector $\left(m_{2}, \ldots, \quad m_{\mathrm{k}}, m_{1}\right)$. Then an encryption of the vector $\left(m_{1}+m_{2}, m_{2}+m_{3}, \ldots, m_{\mathrm{k}}+m_{1}\right)$ is obtained by adding the original ciphertext. We repeatedly apply this method $(\log k-1)$ times with a rotation by a power of two, which generates the desired ciphertext; that is, every plaintext slot contains the same value of $\Sigma_{1 \leq i \leq k} m_{\mathrm{i}}$. The AllSum algorithm is explicitly described in Textbox 1 .

Let us assume that we are given $n$ training data samples $z_{\mathrm{i}}$ with $(d+1)$ features. As mentioned before, our goal is to securely evaluate the following arithmetic circuit:

$$
\beta \leftarrow \beta+(\alpha / n) \Sigma_{1 \leq i \leq n} g\left(-z_{i}^{\mathrm{T}} \beta\right) \cdot z_{i},
$$

where $g(x)$ denotes the approximating polynomial of the sigmoid function chosen in the previous subsection. We set the initial $\beta$ parameters as the zero vector for simplicity.

Because our cryptosystem only supports integer computation, all the elements are scaled by a factor of an integer $p$ and then converted into the nearest integers for quantization. The client first receives the ciphertexts encrypting the vector $\left(p \cdot z_{i}\right)$ from $n$ users, and then compromises them to obtain $(d+1)$ ciphertexts $c t . z_{i}$ for all $j=0,1, \ldots, d$, each of which encrypts the vector $p \cdot\left(z_{1 j}, \ldots, z_{n j}\right)$ of the $j$-th attributes using batching technique. If $n$ is not a power of two, the plaintext slots are zero padded so that the number of slots divides $N / 2$. Finally, these resulting ciphertexts $\left(c t . z_{0}, \ldots, c t . z_{d}\right)$ are sent to the server for the computation of gradient descent. 
Figure 2. Graphs of (a) sigmoid function and Taylor polynomials and (b) sigmoid function and least squares approximations.
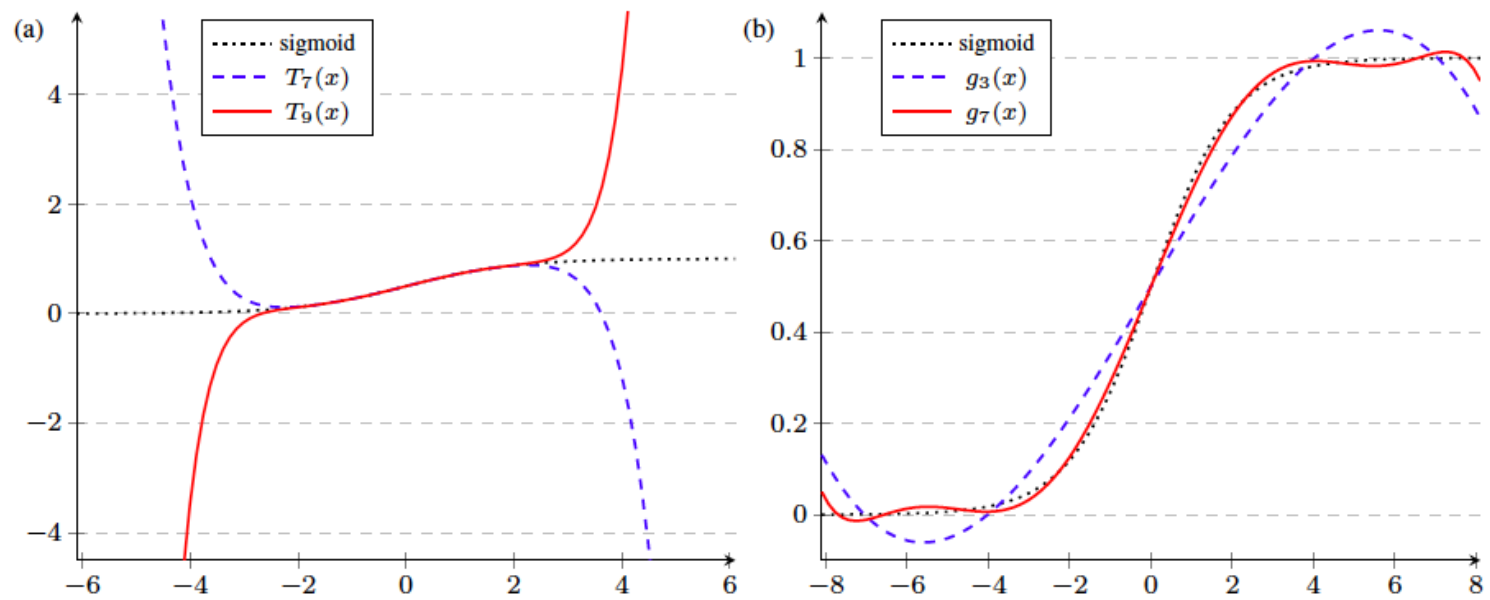

Textbox 1. The AllSum algorithm.

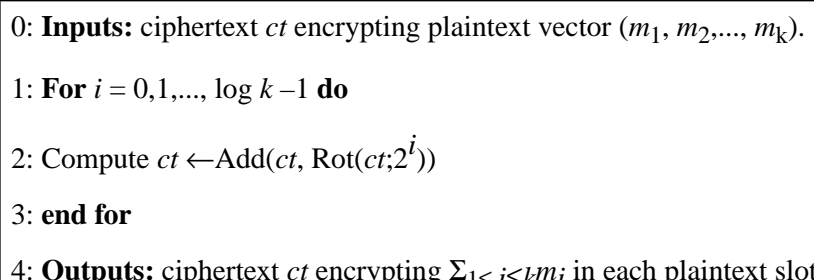

Textbox 2. Secure logistic regression algorithm.

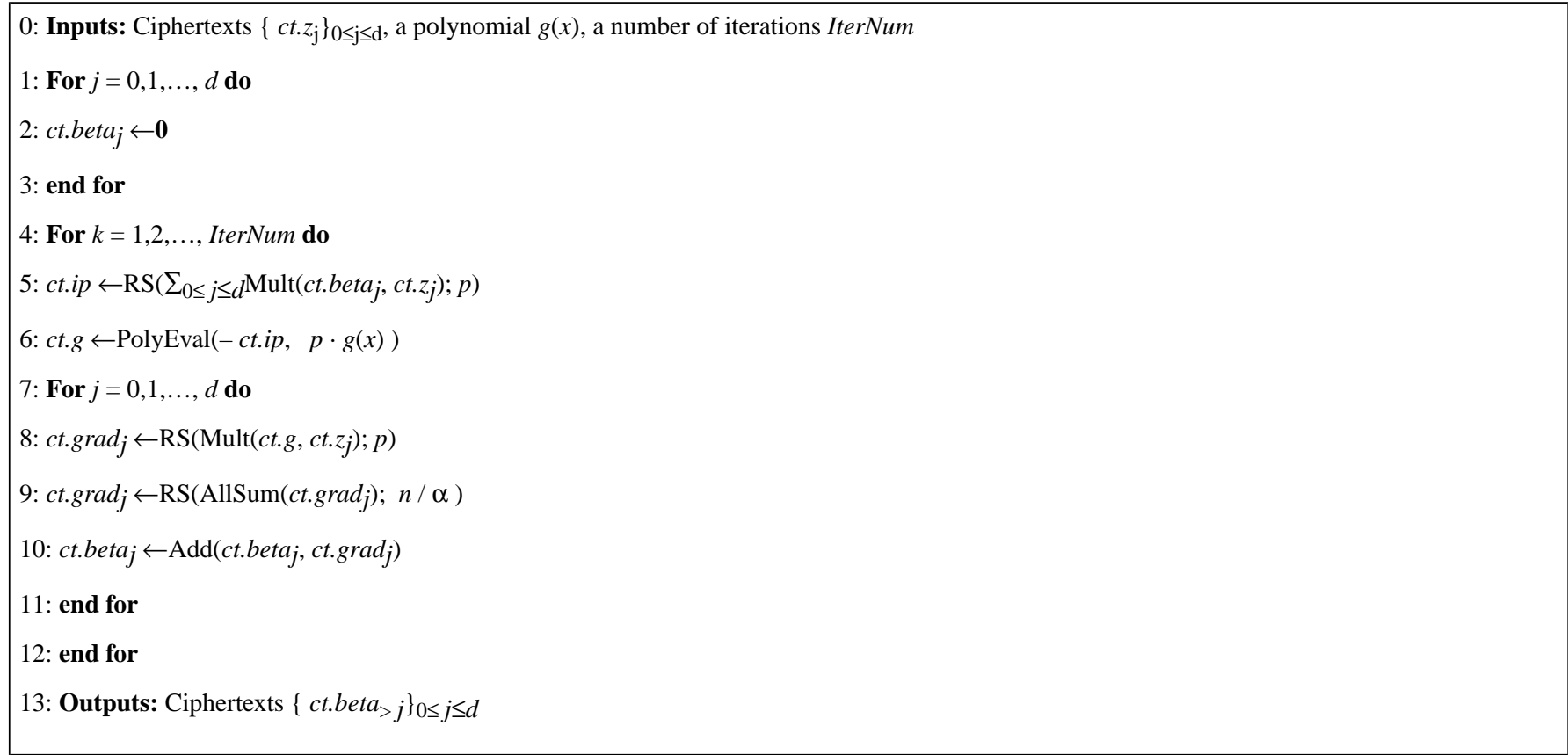

The public server generates the initial ciphertexts $\left(\right.$ ct.beta $_{0}, \ldots$, ct.beta $)$ as zero polynomials in $\mathrm{R}_{q}$ (the residue ring of $\mathrm{R}=\mathrm{Z}[\mathrm{X}] /\left(\mathrm{X}^{N}+1\right)$ modulo an integer $\left.q\right)$. At each iteration, it performs a homomorphic multiplication of ciphertexts $c t . b e t a_{j}$ and $c t . z_{j}$, and outputs a ciphertext encrypting the plaintext vector $p^{2} \cdot\left(z_{1 j}{ }^{\mathrm{T}} \beta_{j}, \ldots, z_{n j}{ }^{\mathrm{T}} \beta_{j}\right)$ for all $j=0, \ldots, d$. Then it aggregates the ciphertexts and performs the rescaling operation with a scaling factor of $p$ to manipulate the size of plaintext, returning a ciphertext $c t$.ip that represents a plaintext vector approximating to $p \cdot\left(z_{1}^{\mathrm{T}} \beta, \ldots, z_{\mathrm{n}}^{\mathrm{T}} \beta\right)$.

For the evaluation of the least squares polynomial $g(x)$ at $\left(-z_{i}^{\mathrm{T}} \beta\right)$, we adapt the polynomial evaluation algorithm, denoted by PolyEval( $\cdot)$, suggested in [18]. Each coefficient of the polynomial should be scaled by a factor of $\mathrm{p}$ to be transformed into an integral polynomial. The output ciphertext $c t$. $g$ contains $p \cdot g\left(-z_{i}^{\mathrm{T}} \beta\right)$ in the $i$-th slot. Finally, the server performs a 
homomorphic multiplication of the ciphertexts $c t . g$ and $c t . z_{j}$, AllSum procedure, and rescaling by a factor of $n / \alpha$ (nearest integer to $n / \alpha)$. These procedures generate ciphertexts ct.grad $0, \ldots, c t$. grad $_{d}$ corresponding to the entries of the gradient vector weighted by the learning rate and the sample size. Then it only needs to perform an addition with the model parameters $\beta$ and the gradient vector over encryption, which yields a new ciphertext ct.beta $_{j}$ that encrypts an approximation of the $j$-th scaled value of the gradient update in Equation 7. Our secure logistic regression algorithm is described in Textbox 2 .

Our solution can compute the gradient descent algorithm securely; however, its direct implementation is not efficient and requires a total ciphertext modulus of $\log p \cdot(\log \operatorname{deg}(g)+3)+\log (n / \alpha)$ bits at each iteration, where $x$ denotes the smallest integer that is not less than $x$. We further optimized this algorithm by manipulating the arithmetic circuit for the update term $(\alpha / n) \Sigma_{1 \leq i \leq n} g\left(-z_{i}^{\mathrm{T}} \beta\right) \cdot z_{i}$ and could reduce the ciphertext modulus to $3 \cdot \log p+\log (n / 4 \alpha)$ bits or $4 \cdot \log p+\log (n / 4 \alpha)$ bits when $g(x)=g_{3}(x)$ or $g(x)=g_{7}(x)$, respectively. Interested readers can refer to Multimedia Appendix 2 for more details.

\section{Results}

\section{Implementation Details}

All experiments were performed on an Intel Xeon running at $2.3 \mathrm{GHz}$ processor with 16 cores and $64 \mathrm{~GB}$ of RAM, which is an m4.4xlarge AWS EC2 instance. In our implementation, we used a variant of a fixed-point homomorphic encryption scheme of Cheon et al [18,24] with C++-based Shoup's Number Theory Library [25]. Our implementation is publicly available at GitHub [26].

\section{Datasets}

We developed our approximation algorithm using the Myocardial Infarction dataset from Edinburgh [27]. The others were obtained from Low Birth Weight Study, Nhanes III, Prostate Cancer Study, and Umaru Impact Study datasets [28-31]. All these datasets have a single binary outcome variable, which can be readily used to train binary classifiers such as logistic regression. Table 2 illustrates the datasets with the number of observations (rows) and the number of features (columns), respectively. We utilized five-fold cross-validation that randomly partitions the original datasets into five folds with the approximately equal size; we used four subsets for learning (with the learning rate $\alpha \approx 1$ ) and one subset for testing the trained model..

\section{Parameters and Timings for the Homomorphic Encryption Scheme}

We assumed that all inputs had $\log p=28$ bits of precision and set the bit length of the output ciphertext modulus as $\log q_{0}=\log p+10$. As discussed previously, when evaluating the gradient descent algorithm with $g(x)=g_{7}(x)$, a ciphertext modulus is reduced more than $g(x)=g_{3}(x)$ at each iteration. Thus, we set the number of iterations as IterNum $=25$ (resp IterNum $=20$ ) when $g(x)=g_{3}(x)$ (resp. $\left.g(x)=g_{7}(x)\right)$ to take an initial ciphertext modulus of similar size. We could actually obtain the approximate bit length of fresh ciphertext modulus $\log q$ around 2204 to 2406 . The parameter set provides 80 bits of security (see Multimedia Appendix 3 for more details). Because all the computations were performed on encrypted data, the security against a semi-honest adversary follows from the semantic security of the underlying homomorphic encryption scheme. For this setting, the size of the public key and a freshly encrypted ciphertext is $75 \mathrm{MB}$. The key generation takes approximately 56 to 58 seconds and the encryption takes approximately 1.1 to 1.3 seconds.

In Table 3, we evaluated our models performance based on average running time (encryption, evaluation, and decryption) and storage (encrypted dataset size) in each fold.

We used a popular metric, area under the receiver operating characteristic curve (AUC), to measure the model's classification performance when the true positive rate was plotted against the false positive rate at various thresholds. Figure 3 plots the average AUC values from five-fold cross-validation for datasets. The program was implemented by MATLAB 2017a.

We can converge to the optimum within a small number of iterations $(20 \sim 25)$, which makes it very promising to train a homomorphically encrypted logistic regression model and mitigate the privacy concerns.

In Table 4, we compared the produced models using our encrypted approach and unencrypted logistic regression. In the unencrypted cases, we used the original sigmoid function on the same training dataset with the same iteration numbers as the encrypted cases. For discrimination, we calculated the accuracy (\%), which is defined by the percentage of the correct predictions on the testing dataset. For a more accurate comparison, we used the MSE that measures the average of the squares of the errors. We could also normalize it by dividing by the average of the squares of the (unencrypted) model parameters, called a normalized mean squared error (NMSE).

Table 2. Description of datasets.

\begin{tabular}{lll}
\hline Dataset & Number of observations & Number of features \\
\hline Edinburgh Myocardial Infarction & 1253 & 10 \\
Low Birth Weight Study & 189 & 10 \\
Nhanes III & 15,649 & 16 \\
Prostate Cancer Study & 379 & 10 \\
Umaru Impact Study & 575 & 9 \\
\hline
\end{tabular}


Table 3. Experiment results of our homomorphic encryption-based logistic regression algorithm

\begin{tabular}{|c|c|c|c|c|}
\hline Dataset and degree of $g(x)$ & Encryption (sec) & Evaluation (min) & Decryption (sec) & Storage (GB) \\
\hline \multicolumn{5}{|c|}{ Edinburgh Myocardial Infarction } \\
\hline 3 & 12 & 131 & 6.3 & 0.69 \\
\hline 7 & 12 & 116 & 6.0 & 0.71 \\
\hline \multicolumn{5}{|l|}{ Low Birth Weight Study } \\
\hline 3 & 11 & 101 & 4.9 & 0.67 \\
\hline 7 & 11 & 100 & 4.5 & 0.70 \\
\hline \multicolumn{5}{|l|}{ Nhanes III } \\
\hline 3 & 21 & 265 & 12 & 1.15 \\
\hline 7 & 21 & 240 & 13 & 1.17 \\
\hline \multicolumn{5}{|l|}{ Prostate Cancer Study } \\
\hline 3 & 11 & 119 & 4.4 & 0.68 \\
\hline 7 & 11 & 100 & 4.5 & 0.70 \\
\hline \multicolumn{5}{|l|}{ Umaru Impact Study } \\
\hline 3 & 10 & 109 & 5.1 & 0.61 \\
\hline 7 & 10 & 94 & 4.3 & 0.63 \\
\hline
\end{tabular}


Figure 3. Average AUC of encrypted logistic regression. FPR: false positive rate; TPR: true positive rate.

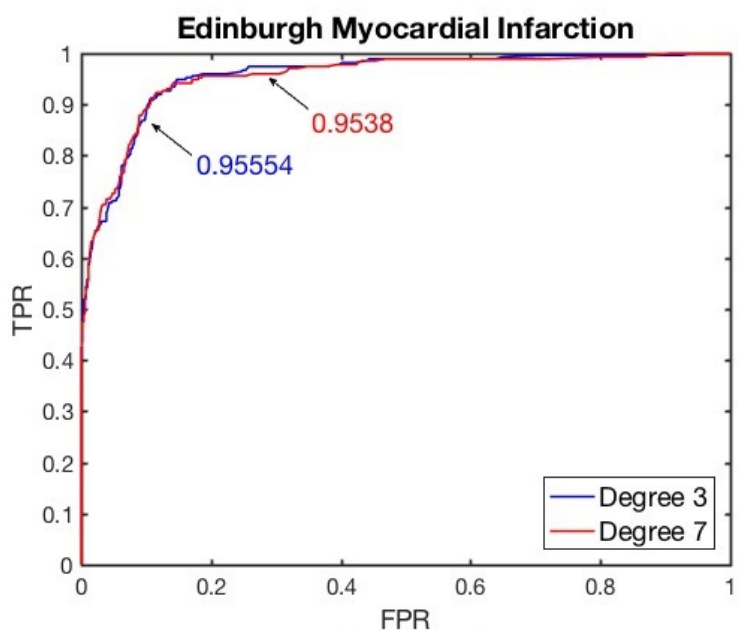

Nhanes III

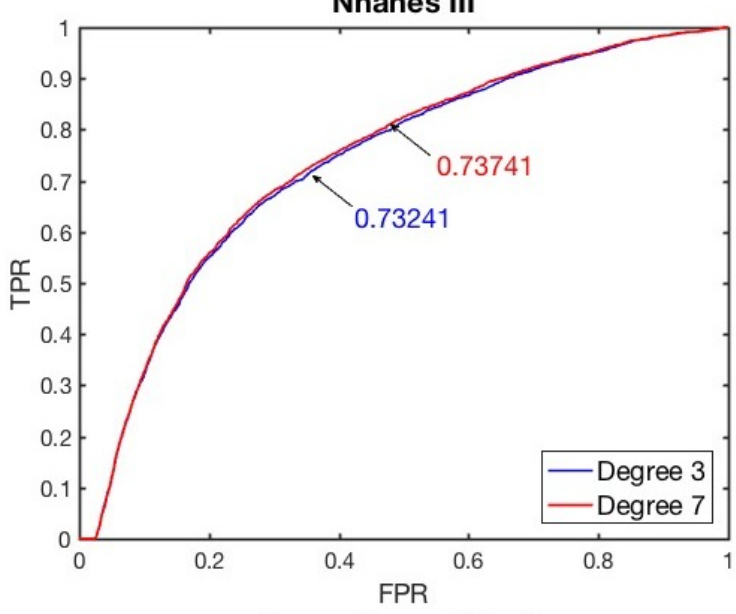

Umaru Impact Study

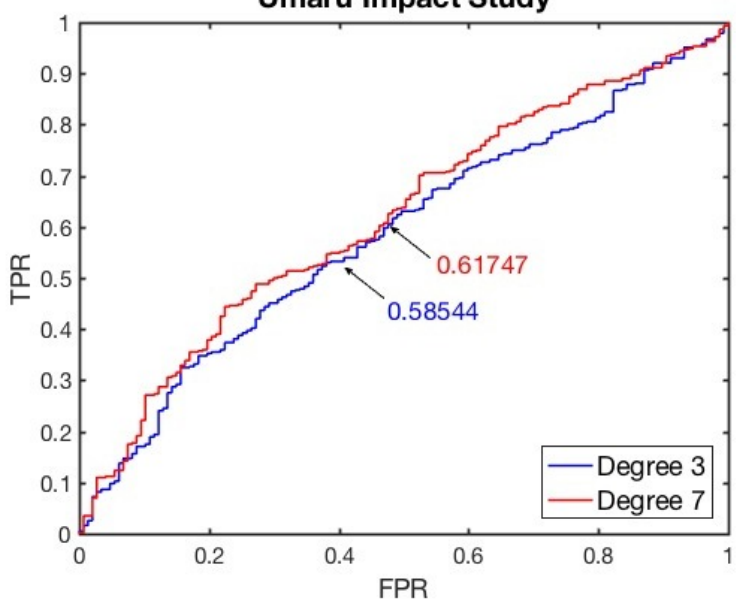

Low Birth Weight Study

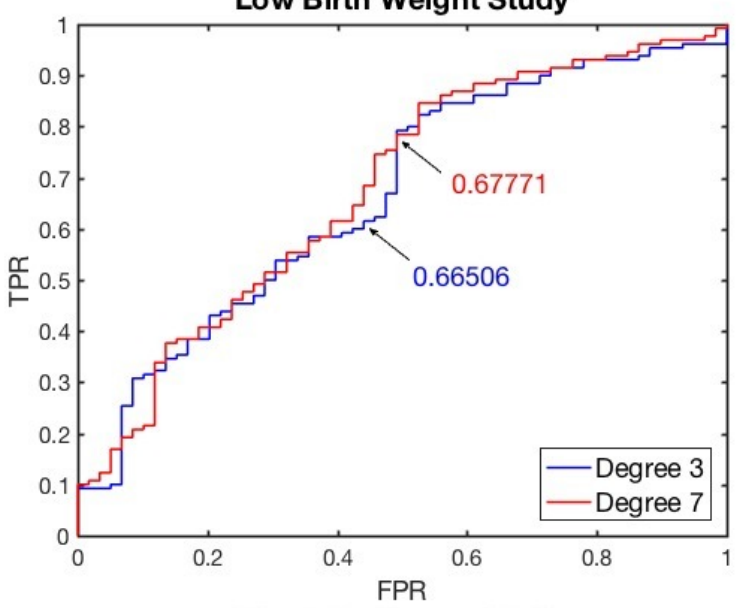

Prostate Cancer Study

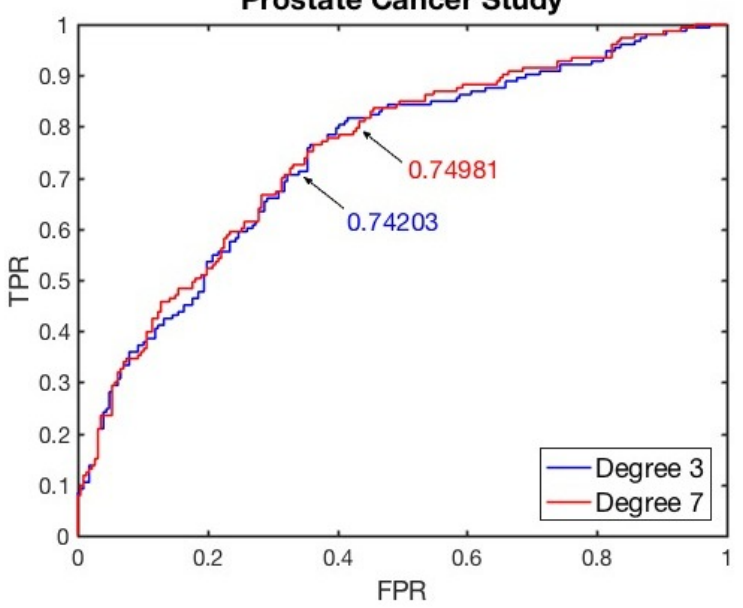


Table 4. Comparison of encrypted/unencrypted logistic regression. AUC: area under the receiver operating characteristic curve. MSE: mean squared error; NMSE: normalized mean squared error.

\begin{tabular}{|c|c|c|c|c|c|c|c|}
\hline \multirow[t]{2}{*}{ Dataset and iteration number } & \multirow[t]{2}{*}{ Degree of $g(x)$} & \multicolumn{2}{|c|}{$\begin{array}{l}\text { Our homomorphic encryption-based } \\
\text { logistic regression }\end{array}$} & \multicolumn{2}{|c|}{ Unencrypted logistic regression } & \multirow[t]{2}{*}{ MSE } & \multirow[t]{2}{*}{ NMSE } \\
\hline & & Accuracy & AUC & Accuracy & AUC & & \\
\hline \multicolumn{8}{|c|}{ Edinburgh Myocardial Infarction } \\
\hline 25 & 3 & $86.03 \%$ & 0.956 & $88.43 \%$ & 0.956 & 0.0259 & 0.0261 \\
\hline 20 & 7 & $86.19 \%$ & 0.954 & $86.19 \%$ & 0.954 & 0.0007 & 0.0012 \\
\hline \multicolumn{8}{|l|}{ Low Birth Weight Study } \\
\hline 25 & 3 & $69.30 \%$ & 0.665 & $68.25 \%$ & 0.668 & 0.0083 & 0.0698 \\
\hline 20 & 7 & $69.29 \%$ & 0.678 & $69.29 \%$ & 0.678 & 0.0003 & 0.0049 \\
\hline \multicolumn{8}{|l|}{ Nhanes III } \\
\hline 25 & 3 & $79.23 \%$ & 0.732 & $79.26 \%$ & 0.751 & 0.0033 & 0.0269 \\
\hline 20 & 7 & $79.23 \%$ & 0.737 & $79.23 \%$ & 0.737 & 0.0002 & 0.0034 \\
\hline \multicolumn{8}{|l|}{ Prostate Cancer Study } \\
\hline 25 & 3 & $68.85 \%$ & 0.742 & $68.86 \%$ & 0.750 & 0.0085 & 0.0449 \\
\hline 20 & 7 & $69.12 \%$ & 0.750 & $69.12 \%$ & 0.752 & 0.0002 & 0.0018 \\
\hline \multicolumn{8}{|l|}{ Umaru Impact Study } \\
\hline 25 & 3 & $74.43 \%$ & 0.585 & $74.43 \%$ & 0.587 & 0.0074 & 0.0829 \\
\hline 20 & 7 & $75.43 \%$ & 0.617 & $74.43 \%$ & 0.619 & 0.0004 & 0.0077 \\
\hline
\end{tabular}

\section{Discussion}

\section{Principal Findings}

Our implementation shows that the evaluation of the gradient descent algorithm with the degree 7 least squares polynomial yields better accuracy and AUC than degree 3. It is quite close to the unencrypted result of logistic regression using the original sigmoid function with the same number of iterations; for example, on the training model of Edinburgh dataset, we could obtain the model parameters $\beta$ as follows:

$$
\begin{aligned}
& (-1.7086,0.0768,0.1119,0.3209,1.2033, \\
& 0.3684,0.9756,0.2020,0.2259,-0.1641),
\end{aligned}
$$

which can reach $86.19 \%$ accuracy and 0.954 AUC on the testing dataset. When using the sigmoid function on the same training dataset, the model parameters $\beta$ are

$$
\begin{aligned}
& (-1.6308,0.0776,0.1097,0.3155,1.1809, \\
& 0.3651,0.9599,0.2083,0.2298,-0.1490),
\end{aligned}
$$

which give the same accuracy and AUC. On the other hand, as shown in Table 4, the MSE and NMSE values of degree 7 are closer to zero which inspires us that the polynomial approximation of that degree is fairly accurate for logistic regression.

One of the inherent properties of our underlying homomorphic encryption scheme is that the inserted errors for security may increase after some homomorphic operations. Hence, the size of error and the precision loss should be discussed carefully to guarantee the correctness of the resulting value. On the other hand, the gradient descent method has a property of negative feedback on computational error. Because we use the gradient at the current weight vector $\beta$ to move it closer to the optimal point of minimized cost, the effect of noise disappears after some iterations. Therefore, there is no need to manage the precision of messages to confirm the correctness of resulting value because the noises are not amplified during evaluation. In our experimentation on the Edinburgh dataset, for instance, the difference between the model parameters obtained from encrypted/unencrypted evaluations was less than $2^{-11}$. This means that we can precisely compute at least most significant 11 bits after the radix point of the model parameters and this approximate vector is accurate enough to achieve a good performance in testing data samples.

\section{Limitations}

There are still a number of limitations in the application of our evaluation model to an arbitrary dataset. First, the use of homomorphic encryption yields the overheads in computation and storage. The size of the dataset should be limited for practical evaluation, but this is not a big problem because there have been significant improvements in the existing homomorphic encryption schemes. The development of homomorphic encryption technology will achieve much better practical performance in our protocol.

Another issue arises from the polynomial approximation. We suggested the least squares method on a certain interval $[-8,8]$, but the precision of the result can increase by managing approximation error from wider range inputs. Finally, our model is based on fixed hyperparameters that should be decided before starting of the evaluation. It would be highly beneficial if we could detect convergence of the loss function in the training process and support early stop instead. 


\section{Conclusions}

This paper presents the first effective methodology to evaluate the learning phase of logistic regression using the gradient descent method based on homomorphic encryption. We have demonstrated the capability of our model across the experiments with different biological datasets. In particular, our solution can be applied to a large-scale dataset, which shows the feasibility of our approach.

\section{Acknowledgments}

The authors would like to thank Kristin Lauter for helpful discussions and suggestions. The authors would also like to thank Andrey Kim for extensive assistance with the code for the homomorphic encryption scheme.

This research of MK, SW, and XJ was supported in part by the National Institute of Health under award numbers R00HG008175, R01GM118574, R01GM118609, and U01EB023685. YS was supported by the National Research Foundation of Korea grant funded by the Korean Government (No: 2017R1A5A1015626).

\section{Authors' Contributions}

MK led the algorithm development and the writing of the methodology. YS, YX, SW, and XJ contributed to the approximation algorithm and evaluation. YS also developed the parallelization for the proposed protocol. XJ and SW motivated the study and blended novel algorithms and new homomorphic schemes to enable secure learning. All authors carefully reviewed and edited the paper.

\section{Conflicts of Interest}

None declared.

\section{Multimedia Appendix 1}

Homomorphic encryption for approximate arithmetic.

[PDF File (Adobe PDF File), 91KB-Multimedia Appendix 1]

\section{Multimedia Appendix 2}

Further optimization of secure logistic regression algorithm.

[PDF File (Adobe PDF File), 128KB-Multimedia Appendix 2]

\section{Multimedia Appendix 3}

How to set parameters.

[PDF File (Adobe PDF File), 65KB-Multimedia Appendix 3]

\section{References}

1. Trinckes Jr J. The Definitive Guide to Complying with the HIPAA/HITECH Privacy and Security Rules. Boca Raton, FL: CRC Press; 2012.

2. Rousseau D. A\&R Translations. 2017 Feb 24. Biomedical research: changing the common rule URL: $\underline{\text { https://www. }}$ ammon-rousseau.com/changing-the-rules-by-david-rousseau/ [accessed 2017-08-19] [WebCite Cache ID 6spHgiYRI]

3. Microsoft. 2016 May 12. Survey results from the Hosting and Cloud Study 2016 URL: https://www.microsoft.com/en-us/ download/details.aspx?id=52045 [accessed 2018-03-04] [WebCite Cache ID 6xg4oxOQh]

4. Wu Y, Jiang X, Kim J, Ohno-Machado L. Grid Binary LOgistic REgression (GLORE): building shared models without sharing data. J Am Med Inform Assoc 2012;19(5):758-764 [FREE Full text] [doi: 10.1136/amiajnl-2012-000862] [Medline: 22511014]

5. Jiang W, Li P, Wang S, Wu Y, Xue M, Ohno-Machado L, et al. WebGLORE: a Web service for Grid LOgistic REgression. Bioinformatics 2013 Dec 15;29(24):3238-3240 [FREE Full text] [doi: 10.1093/bioinformatics/btt559] [Medline: 24072732]

6. Wang S, Jiang X, Wu Y, Cui L, Cheng S, Ohno-Machado L. EXpectation Propagation LOgistic REgRession (EXPLORER): distributed privacy-preserving online model learning. J Biomed Inform 2013 Jun;46(3):480-496 [FREE Full text] [doi: 10.1016/j.jbi.2013.03.008] [Medline: 23562651]

7. Skinner E, Octo Barnett G, Singer D, Mulley A, Lew R, Stickler S, et al. The use of logistic regression in diagnostic and prognostic prediction in a medical intensive care unit. In: Proc Annu Symp Comput Appl Med Care. 1980 Nov 05 Presented at: Proceedings of the Annual Symposium on Computer Application in Medical Care American Medical Informatics Association; ;1; Nov 2-5, 1980; Washington, DC p. 222-227 URL: https://www.ncbi.nlm.nih.gov/pmc/articles/PMC2203702/ 
8. Dietz E. Application of Logistic Regression and Logistic Discrimination in Medical Decision Making. Biometrical J 1987;29(6):747-751 [FREE Full text] [doi: 10.1002/bimj.4710290614]

9. Steyerberg EW, Eijkemans MJ, Harrell FE, Habbema JD. Prognostic modeling with logistic regression analysis: in search of a sensible strategy in small data sets. Med Decis Making 2001;21(1):45-56. [doi: 10.1177/0272989X0102100106] [Medline: 11206946 ]

10. Peduzzi P, Concato J, Kemper E, Holford TR, Feinstein AR. A simulation study of the number of events per variable in logistic regression analysis. J Clin Epidemiol 1996 Dec;49(12):1373-1379. [Medline: 8970487]

11. Graepel T, Lauter K, Naehrig M. ML confidential: machine learning on encrypted data. In: Lecture Notes in Computer Science, vol 7839. Berlin, Heidelberg: Springer; 2012 Presented at: International Conference on Information Security and Cryptology; Nov 28-30, 2012; Seoul p. 1-21 URL: https://link.springer.com/chapter/10.1007\%2F978-3-642-37682-5_1

12. Miotto R, Wang F, Wang S, Jiang X, Dudley JT. Deep learning for healthcare: review, opportunities and challenges. Brief Bioinform 2017 May 06:1. [doi: 10.1093/bib/bbx044] [Medline: 28481991]

13. Dreiseitl S, Ohno-Machado L. Logistic regression and artificial neural network classification models: a methodology review. J Biomed Inform 2002;35(5-6):352-359. [Medline: 12968784]

14. Bos JW, Lauter K, Naehrig M. Private predictive analysis on encrypted medical data. J Biomed Inform 2014 Aug;50:234-243 [FREE Full text] [doi: 10.1016/j.jbi.2014.04.003] [Medline: 24835616]

15. Dowlin N, Gilad-Bachrach R, Laine K, Lauter K, Naehrig M, Wernsing J. CryptoNets: applying neural networks to encrypted data with high throughput and accuracy. In: Proceedings of the 33rd International Conference on International Conference on Machine Learning. 2016 Presented at: 33rd International Conference on Machine Learning; Jun 19-24, 2016; New York p. 201-210.

16. Aono Y, Hayashi T, Trieu PL, Wang L. Scalable and secure logistic regression via homomorphic encryption. In: Proceedings of the Sixth ACM Conference on Data and Application Security and Privacy. 2016 Presented at: Sixth ACM Conference on Data and Application Security and Privacy; Mar 9-11, 2016; New Orleans, LA p. 142-144 URL: https://dl.acm.org/ citation.cfm?doid=2857705.2857731

17. Mohassel P, Zhang Y. SecureML: a system for scalable privacy-preserving machine learning. 2017 Presented at: 2017 IEEE Symposium on Security and Privacy; May 22-26, 2017; San Jose, CA p. 19-38 URL: http://ieeexplore.ieee.org/ document/7958569/

18. Cheon J, Kim A, Kim M, Song Y. Homomorphic encryption for arithmetic of approximate numbers. 2017 Presented at: 23rd International Conference on the Theory and Applications of Cryptology and Information Security; Dec 3-7, 2017; Hong Kong p. 409-437 URL: https://link.springer.com/chapter/10.1007/978-3-319-70694-8 15

19. Ypma T. Historical development of the Newton-Raphson method. SIAM Rev 1995 Dec;37(4):531-551. [doi: $\underline{10.1137 / 1037125]}$

20. Bottou L. Large-scale machine learning with stochastic gradient descent. In: Proceedings of the 19th International Conference on Computational Statistics. 2010 Presented at: 19th International Conference on Computational Statistics; Aug 22-27, 2010; Paris p. 177-186 URL: https://link.springer.com/chapter/10.1007/978-3-7908-2604-3 16

21. Halevi S, Shoup V. Algorithms in HElib. In: Advances in Cryptology - CRYPTO 2014. 2014 Presented at: 34th Annual Cryptology Conference; Aug 17-21, 2014; Santa Barbara, CA p. 554-571.

22. Cheon J, Kim M, Kim M. Search-and-compute on encrypted data. In: International Conference on Financial Cryptography and Data Security. 2015 Presented at: FC 2015 International Workshops; Jan 30, 2015; San Juan, Puerto Rico p. $142-159$.

23. Cheon J, Kim M, Kim M. Optimized Search-and-Compute Circuits and Their Application to Query Evaluation on Encrypted Data. IEEE T Inf Foren Sec 2016 Jan;11(1):188-199 [FREE Full text] [doi: 10.1109/TIFS.2015.2483486]

24. Cheon J, Kim A, Kim M, Song Y. GitHub. 2016. The HEAAN library URL: https://github.com/kimandrik/HEAAN [accessed 2017-08-19] [WebCite Cache ID 6spMzVJ6U]

25. Shoup V. NTL: A Library for doing Number Theory. 2001. URL: http://www.shoup.net/ntl/index.html [accessed 2018-03-04] [WebCite Cache ID 6xgAtJaXs]

26. Kim M, Song Y. GitHub. 2017. Implementation of secure logistic regression based on homomorphic encryption URL: https://github.com/K-miran/HELR [accessed 2017-08-19] [WebCite Cache ID 6spN8xvke]

27. Kennedy RL, Burton AM, Fraser HS, McStay LN, Harrison RF. Early diagnosis of acute myocardial infarction using clinical and electrocardiographic data at presentation: derivation and evaluation of logistic regression models. Eur Heart $\mathbf{J}$ 1996 Aug;17(8):1181-1191. [Medline: $\underline{\text { 8869859] }}$

28. LogisticDx: Diagnostic Tests for Models with a Binomial Response. lbw: Low Birth Weight study data URL: https://rdrr. io/rforge/LogisticDx/man/lbw.html [accessed 2017-08-19] [WebCite Cache ID 6spNFX2b5]

29. LogisticDx: Diagnostic Tests for Models with a Binomial Response. nhanes3: NHANES III data URL: $\underline{\text { https://rdrr.io/rforge/ }}$ LogisticDx/man/nhanes3.html [accessed 2017-08-19] [WebCite Cache ID 6spNJJFDx]

30. LogisticDx: Diagnostic Tests for Models with a Binomial Response. pcs: Prostate Cancer Study data URL: https://rdrr.io/ rforge/LogisticDx/man/pcs.html [accessed 2017-08-19] [WebCite Cache ID 6spNLXr5a]

31. LogisticDx: Diagnostic Tests for Models with a Binomial Response. uis: UMARU IMPACT Study data URL: https://rdrr. io/rforge/LogisticDx/man/uis.html [accessed 2017-08-18] [WebCite Cache ID 6spNOLB9n] 


\section{Abbreviations}

AUC: area under the curve

MSE: mean squared error

NMSE: normalized mean squared error

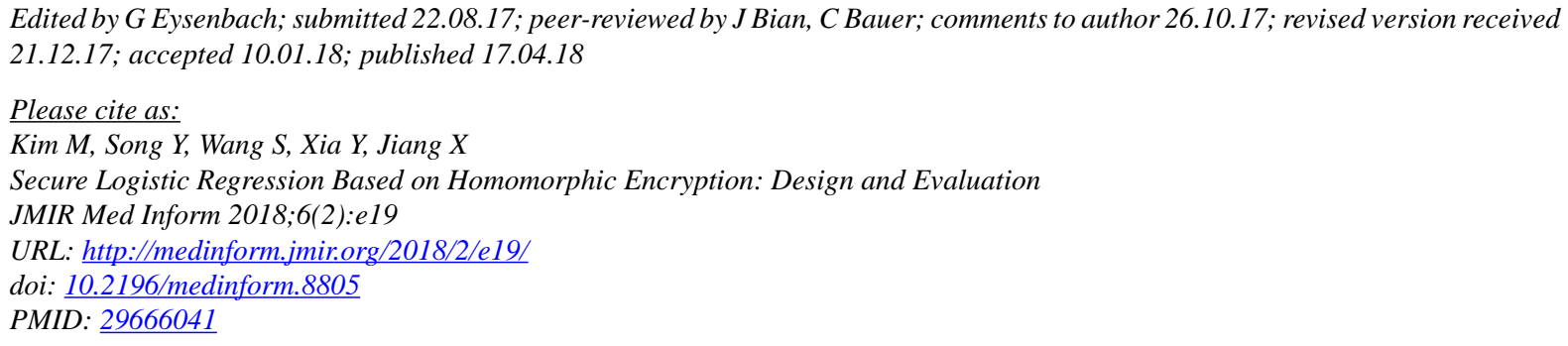

CMiran Kim, Yongsoo Song, Shuang Wang, Yuhou Xia, Xiaoqian Jiang. Originally published in JMIR Medical Informatics (http://medinform.jmir.org), 17.04.2018. This is an open-access article distributed under the terms of the Creative Commons Attribution License (https://creativecommons.org/licenses/by/4.0/), which permits unrestricted use, distribution, and reproduction in any medium, provided the original work, first published in JMIR Medical Informatics, is properly cited. The complete bibliographic information, a link to the original publication on http://medinform.jmir.org/, as well as this copyright and license information must be included. 